\title{
Shallow-Water Theory and Channel Flow with Hydraulic Jump: Non-Steady Consideration
}

\author{
Asuquo E Eyo \\ Department of Mathematics and Statistics, University of Uyo, Uyo, Nigeria \\ E-mail: asuquoessieneyo@yahoo.com
}

Received: August 15, 2011

doi:10.5539/mas.v6n1p50

\author{
Accepted: November 17, $2011 \quad$ Published: January 1, 2012 \\ URL: http://dx.doi.org/10.5539/mas.v6n1p50
}

\begin{abstract}
We examine the classical hydraulic jump in an open channel flow and show that it can be qualitatively understood using the model, namely, equations of shallow-water type. In the analysis, we consider the following cases: (i) unsteady, one dimensional flow of a liquid in an open channel, (ii) uniform and smooth flow with the channel bed flat and viscosity neglected almost everywhere, and (iii) a steady uniform flow U from left (positive flux) with the flow elsewhere taken to be two dimensional. Based on this model the relation for the speed of propagation of the jump in terms of depth ratio is obtained. We employ the method of characteristics and the Riemann invariants to determine the precise location and time of the onset of the hydraulic ump. Our analysis reveals that the location $\mathrm{x}_{\mathrm{J}}$ and time $\mathrm{t}_{\mathrm{J}}$ of the jump formation are influenced by the initial acceleration of the fluid. Specializations are later made to the cases where the initial acceleration is positive and finite, negative, infinite. Results are obtained in each of these cases.
\end{abstract}

Keywords: Equations of shallow-water type, Channel flow, Hydraulic jump, Method of characteristics, Remann inivariants

\section{Introduction}

The rise in water level which occurs during the transformation of the unstable shooting flow to the stable streaming flow is called classical hydraulic jump (Khurmi, 2004). In contrast, the circular hydraulic jump appears when a vertical jet of liquid is directed upon a horizontal plate and spreads out radially in a thin layer. At a certain radius one observes a sudden increase in the height of the liquid the mean position of which does not change with time (Bohr, et al., 1993). On the other hand, the shallow water equations are the simplest form of the equations of motion that can be used to describe the evolution of an incompressible fluid in response to gravitational and rotational accelerations. The solutions of the shallow water equations represent many types of motion, including Rossby waves and inertia-gravity waves. Shallow water waves are approximated by the relation, $c=\sqrt{g h}$, where $\mathrm{c}$ is the local wave speed, $\mathrm{g}$ is the gravitational acceleration and $h$ the depth of water from the bottom to the undisturbed water level; or, are characterized by $\frac{\lambda}{h}>>1$, where $\lambda$ is the wave length and $h$ is as above (Whitham, 1974).

Bohr et al. (1993) discussed circular hydraulic jump using shallow-water equations for the case of stationary flow. Their main conclusion here was that it was not possible to determine the position of the jump from the ideal theory. However, they showed that the jump position could be determined using viscous shallow-water theory by connecting the inner and outer solutions through a shock.

Bowles (1995) studied steady planar flow of a liquid layer over an obstacles for favourable slopes. He found half-plane Poiseuille flow to be a non-unique solution on a uniformly sloping surface since eigensolutions exist which were initially exponentially small far upstream. He also addressed the possibility of standing waves downstream of the jump for various slope magnitudes. By adopting numerical technique he found that numerical solutions agreed favourably with the analytical results.

Didenkulova and Pelinovsky (2010) discussed the problem of transmission of wave energy in strongly inhomogenous media with application to long water wave propagating in a basin with a quartic bottom profile. Using the linear water theory they showed that wave component of the flow disturbance could be described by a 
traveling wave solution with an amplitude and phase that could vary with distance. Conditions for wave breaking in the nearshore were also found from the asymptotic solution of the nonlinear shallow water theory.

Didenkulova et al. (2010) studied tsunami wave generation by submarine landslides of a variable volume in a basin of variable depth within the shallow water theory. They also studied analytically the problem of landslide-induced tsunami wave generation and propagation for two specific convex bottom profiles. In these cases they reduced the basic equations to the constant-coefficient wave equation with the forcing determined by the landslide motion. Finally, they studied the dynamics of the waves propagating off-shore for the case of a near-resonant motion of the landslide along the power bottom profiles using the asymptotic approach. They predicted that if the landslide is moving in the fully resonant regime the explicit formula for the amplitude of the wave could be derived. Hence, they demonstrated that generally tsunami wave amplitude varies non-monotonically with distance.

Didenkulova and Pelinovsky (2011) studied nonlinear wave dynamics of long water waves in an inclined channel of a parabolic cross-section, a situation which occurs when sea waves enter and propagate in a narrow bay or fjord. They showed that nonlinear shallow water equations could in this case be written in one dimensional form and solved analytically with the use of the hodograph transformation. Their approach generalized the well-known carrier-Greespan transformation for long wave runup on a plane beach. In the case of an inclined channel of a parabolic cross-section, it led to the associated spherical symmetrical linear wave equation. Finally, they paid special attention to the wave breaking criterion for waves at the coast, which appeared to provide a condition for applicability for the hodograph transformation. They obtained the wave breaking condition and discussed it for each of the studied problem.

El et al. (2009) considered shallow water flow past a broad bottom ridge, localized in the flow direction, using the framework of the forced Su-Gardner (SG) system, with a primary focus on the transcritical regime when the Froude number of the on coming flow was close to unity. A combination of the local transcritical hydraulic solution over the localized topography was used to discuss various flow regimes depending on the combination of the topography height and the Froude number. Their results confirmed that most of the features of the previously developed description were obtained in the framework of the unidirectional forced Korteweg-de Vries $(\mathrm{Kd} \mathrm{V})$ hold-up qualitatively for finite amplitude waves, while the quantitative description could be obtained in the framework of the bidirectional forced SG system.

In his contribution France (1981) investigated the stability of the hydraulic jump and the effectiveness of the jets over a wide range of operating conditions. He observed that the stabilization of the jump is dependent on a number of parameters but concluded however that the angle of inclination of the jets has the more pronounced effect.

Kalish and Bjorkavag (2010) considered energy conservation properties of weak bores in free surface flows. They thought that the energy loss in the shallow water theory for an undular bore was due to upstream oscillations that could carry away the energy lost at the front of the bore. Using a higher order dispersive model equation, their expectation was confirmed through a quantitative study which showed that there was no energy loss if dispersion was accounted for.

Kang et al. (2009) used a new version of the Stimulating Wave Nearshore (SWAN) model (version 40.51) to stimulate the evolution of directional irregular wave near a single break water with the Wen spectrum adopted as an input spectrum. The wave diffraction effect of irregular wave was tested with the experimental data of single break water and with the stimulated results of $\mathrm{Wu}$ Yan and JOHNSWAP spectrum and input data respectively. By comparing the results in four cases they concluded that the new SWAN model with the Wen spectrum could be well used to stimulate wave diffraction around a single break water.

Pelinovsky and Rodin (2011) investigated the process of nonlinear deformation of a wave in shallow water resulting in its collapse and which admits analytical description in the form of the Riemann wave within the framework of shallow water theory. In their work they focused special attention to the wave shape, its spectrum and the collapse moment. Finally they analyzed the process of formation and evolution of the shock wave on shallow water without restriction on its amplitude within the framework of the nonlinear shallow water equations.

Other contributors to the study of this fascinating phenomenon (hydraulic jump) include notably, Bowles and Smith (1992), Chanson (2008), Gregg et al. (2003), Hornung et al. (1995), Higuera (1994), etc. 
In this paper the sole objective is to discuss classical hydraulic jump by means of shallow water equations with a view to determining the exact location and time of the onset of the hydraulic jump. Using the method of characteristics together with the Riemann invariants we obtain the result generally and also in particular cases.

In our analysis we differ from Bohr et al. (1993) in the sense that (i) we are examining the classical hydraulic jump, (ii) we are considering a non-stationary (time-dependent) flow, but using a corresponding shallow-water equations and (iii) we are employing the method of characteristics and Riemann invariants rather than the approximation method adopted by Bohr et al. (1993).

For convenience we divide the flow in the channel into three regions shown in Figure 1.

Region 1 is known as the constant state in which the flow is uniform, steady or constant. Region II is the region in which the fluid speed $\mathrm{u}$ is non-constant and is referred to as the disturbed state, and Region III in which the fluid is at rest $(\mathrm{u}=0)$ is also a constant state.

\section{Canonical (characteristic) Forms of the Shallow Water Equations.}

The governing equations are the equation of the continuity and the momentum equation given respectively by

and

$$
\frac{\partial h}{\partial t}+\frac{u \partial h}{\partial x}+\frac{h \partial u}{\partial x}=0
$$

$$
\frac{\partial u}{\partial t}+\frac{u \partial u}{\partial x}+\frac{g \partial h}{\partial x}=0
$$

where $\mathrm{u}=$ horizontal component of fluid velocity, $\mathrm{h}=$ water depth, $\mathrm{g}=$ gravitational acceleration and $\mathrm{x}$ and $\mathrm{t}$ are the space and time coordinates respectively.

In matrix notation, the system (1) and (2) becomes

or

$$
\left(\begin{array}{l}
h_{t} \\
u_{t}
\end{array}\right)+\left(\begin{array}{ll}
u & h \\
g & u
\end{array}\right)\left(\begin{array}{l}
h_{x} \\
u_{x}
\end{array}\right)=\left(\begin{array}{l}
0 \\
0
\end{array}\right)
$$

$$
u_{t}+A u_{x}=0
$$

where

The matrix A has the eigenvalues

$$
A=\left(\begin{array}{ll}
u & h \\
g & u
\end{array}\right), \quad u_{t}=\left(\begin{array}{l}
h_{t} \\
u_{t}
\end{array}\right) \quad u_{x}=\left(\begin{array}{l}
h_{x} \\
u_{x}
\end{array}\right)
$$

$$
\lambda_{1}=u-\sqrt{g h}, \quad \lambda_{2}=u+\sqrt{g h}
$$

Eigenvector corresponding to $\lambda_{1}$ is $u^{(1)}$ where

$$
u^{(1)}=\left(\begin{array}{c}
h \\
-\sqrt{g h}
\end{array}\right)
$$

The eigenvector corresponding to $\lambda_{2}$ is

Thus, the fundamental matrix $\mathrm{P}$ becomes

$$
u^{(2)}=\left(\begin{array}{c}
h \\
\sqrt{g h}
\end{array}\right)
$$

$$
P=\left(\begin{array}{cc}
h & h \\
-\sqrt{g h} & \sqrt{g h}
\end{array}\right)
$$

so that

Now

$$
P^{-1}=\left(\begin{array}{cc}
\frac{1}{2 h} & \frac{-1}{2 \sqrt{g h}} \\
\frac{1}{2 h} & \frac{1}{2 \sqrt{g h}}
\end{array}\right)
$$

$$
\mathrm{P}^{-1} \mathrm{AP}=\wedge=\text { diagonal matrix }
$$


ie.

which simplifies to

$$
\left(\begin{array}{cc}
\frac{1}{2 h} & \frac{-1}{2 \sqrt{g h}} \\
\frac{1}{2 h} & \frac{1}{2 \sqrt{g h}}
\end{array}\right)\left(\begin{array}{ll}
u & h \\
g & u
\end{array}\right)\left(\begin{array}{cc}
h & h \\
-\sqrt{g h} & \sqrt{g h}
\end{array}\right)=\Lambda
$$

$$
\Lambda=\left(\begin{array}{cc}
u-\sqrt{g h} & 0 \\
0 & u+\sqrt{g h}
\end{array}\right)
$$

From (11) we find

$$
\mathrm{A}=\mathrm{P} \Lambda \mathrm{P}^{-1}
$$

Substituting (12) into the system (4) we get

$$
\left(\begin{array}{l}
\frac{\partial h}{\partial t} \\
\frac{\partial u}{\partial t}
\end{array}\right)+P \Lambda P^{-1}\left(\begin{array}{l}
\frac{\partial h}{\partial x} \\
\frac{\partial u}{\partial x}
\end{array}\right)=0
$$

i.e

i.e

$$
P^{-1}\left(\begin{array}{l}
\frac{\partial h}{\partial t} \\
\frac{\partial u}{\partial t}
\end{array}\right)+\Lambda\left[P^{-1}\left(\begin{array}{l}
\frac{\partial h}{\partial x} \\
\frac{\partial u}{\partial x}
\end{array}\right)\right]=0
$$

$$
\left(\begin{array}{cc}
\frac{1}{2 h} & \frac{-1}{2 \sqrt{g h}} \\
\frac{1}{2 h} & \frac{1}{2 \sqrt{g h}}
\end{array}\right)\left(\begin{array}{c}
\frac{\partial h}{\partial t} \\
\frac{\partial u}{\partial t}
\end{array}\right)+\left(\begin{array}{cc}
u-\sqrt{g h} & 0 \\
0 & u+\sqrt{g h}
\end{array}\right)\left(\begin{array}{cc}
\frac{1}{2 h} & \frac{-1}{2 \sqrt{g h}} \\
\frac{1}{2 h} & \frac{1}{2 \sqrt{g h}}
\end{array}\right)\left(\begin{array}{l}
\frac{\partial h}{\partial x} \\
\frac{\partial u}{\partial x}
\end{array}\right)=0
$$

Equation (14) reduces to

$$
\begin{aligned}
& \frac{1}{2 h} \frac{\partial h}{\partial t}-\frac{1}{2 \sqrt{g h}} \frac{\partial u}{\partial t}+(u-\sqrt{g h})\left(\frac{1}{2 h} \frac{\partial h}{\partial x}-\frac{1}{2 \sqrt{g h}} \frac{\partial u}{\partial x}\right)=0 \\
& \frac{1}{2 h} \frac{\partial h}{\partial t}-\frac{1}{2 \sqrt{g h}} \frac{\partial u}{\partial t}+(u+\sqrt{g h})\left(\frac{1}{2 h} \frac{\partial h}{\partial x}+\frac{1}{2 \sqrt{g h}} \frac{\partial u}{\partial x}\right)=0
\end{aligned}
$$

Taking the local wave speed for long waves on shallow water

$$
c=\sqrt{g h}
$$

as one of the variables, instead of $h$ and still retaining $u$ as the other variable, we then have $u$ and $c$ as our independent variables of the system, each of which depends on $(\mathrm{x}, \mathrm{t})$. From (17) we find

$$
h=\frac{c^{2}}{g}
$$

so that substituting (18) into (15) and (16) and simplifying we find respectively

and

$$
2 \frac{\partial c}{\partial t}-\frac{\partial u}{\partial t}+(u-c)\left(2 \frac{\partial c}{\partial x}-\frac{\partial u}{\partial x}\right)=0
$$

$$
2 \frac{\partial c}{\partial t}+\frac{\partial u}{\partial t}+(u+c)\left(2 \frac{\partial c}{\partial x}+\frac{\partial u}{\partial x}\right)=0
$$


In term of differential operator (19) and (20) can be expressed respectively in the following form

$$
\begin{aligned}
& \left\{\frac{\partial}{\partial t}+(u-c) \frac{\partial}{\partial x}\right\}(u-2 c)=0 \\
& \left\{\frac{\partial}{\partial t}+(u+c) \frac{\partial}{\partial x}\right\}(u+2 c)=0
\end{aligned}
$$

which are the canonical forms (or the characteristic forms) of the shallow-water equations (1) and (2).

\section{Introduction of Riemann Invariants}

The two wave propagation speeds are $\mathrm{u} \pm \mathrm{c}$; that is, the sum of the fluid speed and wave speed as well as the difference of the fluid speed and wave speed. Thus, the new Riemann invariants $\mathrm{r}$ and $\mathrm{s}$ are given by

$$
\begin{gathered}
r(\eta)=u+2 c \\
s(\xi)=u-2 c
\end{gathered}
$$

and hence $r$ and s satisfy

$$
\begin{aligned}
& \left\{\frac{\partial}{\partial t}+(u+c) \frac{\partial}{\partial x}\right\} r=0 \\
& \left\{\frac{\partial}{\partial t}+(u-c) \frac{\partial}{\partial x}\right\} s=0
\end{aligned}
$$

Here $\xi$ and $\eta$ are the characteristic coordinates. Thus

$$
r(\eta)=u+2 c
$$

is constant along $\mathrm{C}^{(1)}$ characteristic (positive characteristic) with slope

$$
\frac{d x}{d t}=u+c
$$

Similarly,

$$
s(\xi)=u-2 c
$$

is constant along $\mathrm{C}^{(2)}$ characteristic (negative characteristic) with slope

$$
\frac{d x}{d t}=u-c
$$

Now, from (23) and (24) we find

$$
\begin{gathered}
u=\frac{r+s}{2} \\
c=\frac{r-s}{4}
\end{gathered}
$$

showing that it is possible to express the dependent variables $u$ and $\mathrm{c}$ in terms of the Riemann invariants.

\section{Location and Time of the Onset of the Jump}

The one-dimensional unsteady problem involves determining the fluid motion induced by a steady uniform flow $\mathrm{U}$ from left in an open channel with horizontal bed. In what follows, the initial region known as a constant state in which the flow is steady and uniform is denoted by $\mathrm{I}$ (Figure 1), so that $\mathrm{u}=\mathrm{U}$ at $\mathrm{t}=0$ and the local wave speed $\mathrm{c}=\mathrm{c}_{0}$. After the origin $\mathrm{O}$ (Figure 1) is the next region denoted by II in which the fluid moves with speed $\mathrm{u}$ at time $\mathrm{t}$ and the wave speed $c=\sqrt{g h}$. This region is referred to as a disturbed state since $\mathrm{u}$ is non-constant here. The third region known as a constant state is a region of fluid at rest and is denoted by III, so that $\mathrm{u}=0$ and $\mathrm{c}=$ $\mathrm{c}_{0}$. Hence region II connects two different constant state regions I and III.

The positive characteristic $C_{0}^{(1)}$ that bounds region III passes through the point L (Figure 2) and is obtained by integrating

so that $C_{0}^{(1)}$ characteristic has the equation.

$$
\frac{d x}{d t}=(u+c)_{0}=c_{0}=\text { constant }
$$

$$
x=c_{0} t
$$

Region II is traversed by a family of straight line characteristics $\mathrm{C}^{(1)}$, each of which issues out from a point between $\mathrm{O}$ and $\mathrm{L}$ shown in Figure 2. 
The slope of a typical member of these characteristics through point $\mathrm{S}$ will be the sum of the wave speed $\mathrm{c}$ and the fluid speed $\mathrm{u}$ at $\mathrm{S}$ given by (28)

$$
\frac{d x}{d t}=(u+c)
$$

All the $\mathrm{C}^{(1)}$ characteristics originating from points between $\mathrm{O}$ and $\mathrm{L}$ will converge in region II and form an envelope starting at point $\mathrm{J}$ shown in Figure 3.

Point $\mathrm{J}$ therefore represents the start of a hydraulic jump, across which the fluid velocity will be discontinuous (Figure 3).

We will now determine the precise location of the point $\mathrm{J}$ on the characteristic LV given by $C_{0}^{(1)}$ with equation $\mathrm{x}=\mathrm{c}_{0} \mathrm{t}$ (Figure 3). To carry out this we invoke the Riemann invariants (23) and (24)

$$
\begin{aligned}
& r(\eta)=u+2 c \\
& s(\xi)=u-2 c
\end{aligned}
$$

The family of straight line characteristics $\mathrm{C}^{(1)}$ through $\mathrm{O}$ that traverse region II have the equation

$$
\frac{\underline{x}}{t}=\zeta
$$

where $\zeta$ is a parameter, with $C_{0}^{(1)}$ corresponding to $\zeta=\mathrm{c}_{0}$. A particular member of this family of characteristics is represented by the dotted line shown in Figure 3. We note that all the $\mathrm{C}^{(2)}$ characteristics must enter the constant state region III in which $u=0, c=c_{0}$, so that the Riemann invariant $s(\xi)$ in (24) must be identically constant and of the form

or

$$
u-2 c=-2 c_{0}
$$

$u=2\left(c-c_{0}\right)$

But since, from (35), $\frac{d x}{d t}=\zeta$ along $\mathrm{C}^{(1)}$ characteristics which are themselves defined by (28)

we have

$$
\frac{d x}{d t}=u+c,
$$

$$
\zeta=u+c
$$

so that elimination of $\mathrm{c}$ in (36) using (37) then gives

Also, elimination of $\mathrm{u}$ between (36) and (38) yields

$$
u=\frac{2}{3}\left(\zeta-c_{0}\right)
$$

$$
c=\frac{1}{3}\left(\zeta+2 c_{0}\right)
$$

and between (35), (36) and (39) gives a complete solution to our problem. In terms of $\mathrm{x}$ and $\mathrm{t}$ we obtain

and

$$
u=\frac{2}{3}\left(\frac{x}{t}-c_{0}\right)
$$

$$
c=\frac{1}{3}\left(\frac{x}{t}+2 c_{0}\right)
$$

which specify respectively $\mathrm{u}$ and $\mathrm{c}$ at points in region II of Figure 2.

We wish now to determine the location of the point $\mathrm{J}$ at which a hydraulic jump first forms on the advancing wave front $C_{0}^{(1)}$ when the stream advances into the channel. Let the coordinates of $\mathrm{J}$ be $\left(\mathrm{x}_{\mathrm{J}}, \mathrm{t}_{\mathrm{J}}\right)$. It follows directly from (38) and (39) that

$$
c=c_{0}+\frac{1}{2} u
$$

Thus, equation (42) relates $\mathrm{c}$ throughout region II to the single variable u. To find out how u varies, we now substitute $\mathrm{c}$ of equation (42) into the continuity equation (21) to get the equation satisfied by $\mathrm{u}$ :

$$
\frac{\partial u}{\partial t}+\left(c_{0}+\frac{3 u}{2}\right) \frac{\partial u}{\partial x}=0
$$

Equation (43) is therefore equivalent to the pair of ordinary differential equations

$$
\frac{d u}{d t}=0 \quad \text { along the curves } \quad \frac{d x}{d t}=c_{0}+\frac{3 u}{2}
$$

so that $\mathrm{u}=$ constant along members of the family of the characteristic curves that are obtained by integrating the second equation in (44). 
Now, suppose the pathline (path of the fluid particle) in the $(\mathrm{x}, \mathrm{t})$ plane is given in terms of $\mathrm{t}$ by the equation.

$$
x=\theta(t)
$$

with the initial conditions

$$
\theta(0)=0 \quad \text { and } \quad\left(\frac{d \theta}{d t}\right)_{t=0}=\theta^{\prime}(0)=0
$$

So that the fluid particle starts from rest at the origin. [Here' denotes differentiation with respect to time]. Let the coordinate of the point $S$ (Figure 3 ) be $(\theta(\tau), \tau)$. Then the velocity of flow here will be $\theta^{\prime}(\tau)$. But, as $u=$ constant along the characteristics of equation (43), and also $u$ equals the fluid speed at $S$, then the equation of the straight line characteristic through $\mathrm{S}$ is obtained by integrating the second equation of (44). Thus, we find

$$
x=c_{0} t+\frac{3}{2} \theta^{\prime}(\tau) t+A
$$

where A is the constant of integration and $u$ in $(44)$ is $\theta^{\prime}(\tau)$. Since this line (47) passes through $S$, the point $(\theta(\tau)$, $\tau)$ satisfies it. Thus

$$
\theta(\tau)=c_{0} \tau+\frac{3}{2} \theta^{\prime}(\tau) \tau+A
$$

where

$$
A=\theta(\tau)-\left\{c_{0}+\frac{3}{2} \theta^{\prime}(\tau)\right\} \tau
$$

Substituting (49) into (48) and factoring we find

$$
x=\theta(\tau)+\left\{c_{0}+\frac{3}{2} \theta^{\prime}(\tau)\right\}(t-\tau)
$$

as the required equation of the straight line through S. Differentiating (50) partially with respect to $\tau$ yields

whence

$$
0=\theta^{\prime}(\tau)+\frac{3}{2} \theta^{\prime \prime}(\tau)(t-\tau)-c_{0}-\frac{3}{2} \theta^{\prime}(\tau)
$$

$$
\theta^{\prime \prime}(\tau)=\frac{2 c_{0}+\theta^{\prime}(\tau)}{3(t-\tau)}
$$

Since we are interested only in the location of the start of the jump at $\mathrm{J}$ in the characteristic $C_{0}^{(1)}$ through the origin, we require to take the point $S$ to be the origin. This is equivalent to setting $\tau=0$ in (50), and using the initial conditions (46) we obtain

$$
x_{J}=c_{0} t_{J}
$$

Similarly setting $\tau=0$ in (52) and applying the same conditions (46) we obtain

$$
\theta^{\prime \prime}(\tau)=\frac{2 c_{0}}{3 t_{J}}
$$

which is the initial acceleration of the fluid. Consequently

Substitution of (55) into (53) gives

$$
t_{J}=\frac{2 c_{0}}{3 \theta^{\prime \prime}(0)}
$$

$$
x_{J}=\frac{2 c_{0}^{2}}{3 \theta^{\prime \prime}(0)}
$$

Thus (56) and (55) give respectively the precise location and time of the onset of the hydraulic jump.

\section{Jump Conditions}

Equations (1) and (2) of the shallow-water equations can be re-written in the following conservation forms:

Mass:

$$
\frac{\partial h}{\partial t}+\frac{\partial(u h)}{\partial x}=0
$$

Momentum:

$$
\frac{\partial(u h)}{\partial t}+\frac{\partial}{\partial x}\left(u^{2} h+1 / 2 g h^{2}\right)=0
$$

Energy:

$$
\frac{\partial}{\partial t}\left(1 / 2 u^{2} h+1 / 2 g h^{2}\right)+\frac{\partial}{\partial x}\left(1 / 2 u^{3} h+u g h^{2}\right)=0
$$

As a conventional notation, we use the subscript 1 for the values ahead of the jump (upstream conditions) and the subscript 2 for the values behind it (downstream conditions). Then, if $\bar{\beta}$ is the jump speed, then the jump condition from equation (57) is given as

$$
\bar{\beta}\left(h_{2}-h_{1}\right)=u_{2} h_{2}-u_{1} h_{1}
$$


This condition may also be written in the form

$$
\bar{\beta}[h]=[u h]
$$

Similarly, the jump condition from equation (58) is

$$
\bar{\beta}[u h]=\left\lfloor u^{2} h+1 / 2 g h^{2}\right\rfloor
$$

Thus across a jump (or bore) moving with speed $\bar{\beta}$ the jump conditions are given by (61) and (62). We shall now proceed to determine the relation for the speed $\bar{\beta}$ of propagation of the jump in terms of depth scales. To do this, we write the above jump conditions in terms of

$$
\bar{u}=u-\bar{\beta}
$$

Where $\bar{u}$ is the fluid speed relative to an observer moving with the jump at the speed $\bar{\beta}$. Thus, in terms of (63) the jump conditions (61) and (62) become respectively

and

$$
[\bar{u} h]=0
$$

which we can write again respectively as

$$
\left\lfloor\bar{u} u h+1 / 2 g h^{2}\right\rfloor=0
$$

and

$$
\bar{u}_{1} h_{1}=\bar{u}_{2} h_{2}
$$

$\bar{u}_{1} u_{1} h_{1}+1 / 2 g h_{1}^{2}=\bar{u}_{2} u_{2} h_{2}+1 / 2 g h_{2}^{2}$

As the speed $\bar{\beta}$ of the jump must be continuous across the jump then we must have that

Now, with the aid of (68) equation (67) becomes

$$
[\bar{u}]=[u]
$$

From (66) we have

$$
\left(\bar{u}_{1}\right)^{2} h_{1}+1 / 2 g h_{1}^{2}=\left(\bar{u}_{2}\right)^{2} h_{2}+1 / 2 g h_{2}^{2}
$$

Substituting (70) into (69) and simplifying we find

$$
\bar{u}_{2}=\frac{\bar{u}_{1} h_{1}}{h_{2}}
$$

Writing (63) as

$$
\left(\bar{u}_{1}\right)^{2}=1 / 2 g h_{2} \frac{\left(h_{2}+h_{1}\right)}{h_{1}}
$$

equation (71) becomes

$$
\bar{u}_{1}=u_{1}-\bar{\beta}
$$

giving

$$
\begin{aligned}
& \left(u_{1}-\bar{\beta}\right)^{2}=1 / 2 g h_{2} \frac{\left(h_{2}+h_{1}\right)}{h_{1}} \\
& \left(u_{1}-\bar{\beta}\right)=\left(1 / 2 g h_{2} \frac{\left(h_{2}+h_{1}\right)}{h_{1}}\right)^{1 / 2}
\end{aligned}
$$

Here we consider only the positive root sign since the jump is moving from right to left. Since the jump moves into water of depth $h_{1}$ which is at rest then the upstream speed $u_{1}=0$. Substituting this into (74) the jump speed $\bar{\beta}$ becomes

$$
\bar{\beta}=-\left(1 / 2 g h_{2} \frac{\left(h_{2}+h_{1}\right)}{h_{1}}\right)^{1 / 2}
$$

where $\mathrm{h}_{2}$ is the water depth behind the jump (i.e. downstream depth). Consequently, the water speed $\mathrm{u}_{2}$ behind the jump (i.e. downstream speed) becomes, using (63)

$$
u_{2}=\bar{u}_{2}+\bar{\beta}
$$

which, by using (70) and (72), reduces to

Thus,

$$
u_{2}=\bar{\beta}\left(1-\frac{h_{1}}{h_{2}}\right) \quad\left(\text { since } \mathrm{u}_{1}=0\right)
$$

$$
\bar{\beta}=\frac{u_{2}}{\left(1-\frac{h_{1}}{h_{2}}\right)}
$$

It terms of depth ratio, $H=\frac{h_{2}}{h_{1}}$ (78) becomes

$$
\bar{\beta}=\frac{u_{2}}{\left(1-\frac{1}{H}\right)}
$$


which is the relation for the speed of propagation of the fluid in terms of depth ratio.

\section{Conclusion}

In this work we observe that the occurrence of the jump is influenced mostly by the initial acceleration $\theta^{\prime \prime}(0)$ of the fluid given by (54). Hence we deduce that (i) if the initial acceleration $\theta^{\prime \prime}(0)$ is positive and finite the jump will form at a finite time $\mathrm{t}_{\mathrm{J}}$ given by (55) and at the location $\mathrm{x}_{\mathrm{J}}$ given by (56) after the start of the fluid motion; (ii) if the initial acceleration is negative then no jump will form, that is, there will be a reversed flow; and (iii) if this acceleration is infinite then the result is that the jump will concentrate at the origin as can be observed from (55) and (56). Equations (61) and (62) give the jump (or discontinuity) conditions which were derived from the conservation forms (57) and (58) of the shallow-water equations (1) and (2). The negative sign in (75) indicates that the jump is travelling from right to left to the water of depth $h_{1}$ which is at rest, so that $\mathrm{u}_{1}=0$. The relation for the speed $\bar{\beta}$ of propagation of the jump in terms of depth ratio is given by (79). This relation shows that $\bar{\beta}$ is in direct proportion to downstream speed $\mathrm{u}_{2}$ of the jump. It is pertinent to note that energy is not conserved across the jump since hydraulic jump dissipates energy into turbulence from which it is eventually converted into heat. Hence the jump condition corresponding to (59) is not applicable here since it is not a conservation law.

\section{Acknowledgement}

The author is grateful to the unknown referees for their useful comments and suggestions.

\section{References}

Bhor, T., Dimond, P., \& Putkaradize, V. (1993). Shallow Water Approach to Circular Hydraulic Jump. Journal of Fluid Mechanics, 254, 635-645. http://dx.doi.org/10.1017/S0022112093002289

Bowles, R. L. (1995). Upstream Influence and the Form of Standing Hydraulic Jump in Liquid - Layer Flows on Favourable slopes. Journal of fluid Mechanics, 284, 63-96. http://dx.doi.org/10.1017/S0022112095000279

Bowles, R. L., \& Smith, F. T. (1992). The Standing Hydraulic Jump: Theory, Computations and Comparison with Experiments. Journal of Fluid Mechanics, 242, 145-168. http://dx.doi.org/10.1017/S0022112092002313

Chanson, H. C. (2008). Application of the Saint-Venant Equations and Method of Characteristics on the Dam Break Wave Problem. Report No. CH55/05H.chanson/JAFM, 1, 1-10.

Didenkulova, I., \& Pelinovsky, E. (2010). Travelling Water Waves along a Quartic Bottom Profile. Proceedings of the Estonian Academy of Sciences, 59 (2), 166 - 171. http://dx.doi.org/10.3176/proc.2010.2.16

Didenkulova, I., Nikolkina, I., Pelinovsky, E., \& Zahibo, N. (2010). Tsunami Wave Generated by Submarine Landslides of Variable Volume: Analytical solutions for a Basin of Variables Depth. Natural Hazards and Earth System Sciences, 10, 2407-2419. http://dx.doi.org/10.5194/nhess-10-2407-2010

Didenkulova, I., \& Pelinovsky, E. (2011). Nonlinear Wave Evolution and Runup in an Inclined Channel of a Parabolic Cross-Section. Physics of Fluids, 23 (8), 086602. http://dx.doi.org/10.1063/1.3623467

El, G. A., Grinshaw, R. H. J., \& Smyth, N. F. (2009). Transcritical Shallow Water Flow Past Topography: Finite Amplitude Theory. Journal of Fluid Mechanics, 640, 187 - 214. http://dx.doi.org/10.1017/S0022112009991315

France, P. W. (1981). An Investigation of a Jet-Assisted Hydraulic Jump. Journal of Hydraulic Research, 19, 321 - 337. http://dx.doi.org/10.1080/00221688109499507

Gregg, M. C., Sanford, T. B., \& Winkel, D. P. (2003). Reduced Mixing of the Breaking of Internal Waves in Equatorial Waters. Nature, 422, 513 - 515. http://dx.doi.org/10.1038/nature01507

Hornung, H. G., Wilert, C. E., \& Turner, S. (1995). The Flow Downstream of a Hydraulic Jump. Journal of fluid Mechanics, 287, 299 - 316. http://dx.doi.org/10.1017/S0022112095000966

Huguera, F. J. (1994). The Hydraulic Jump in a Viscous Laminar Flow. Journal of fluid Mechanics, 274, 69 92. http://dx.doi.org/10.1017/S0022112094002041

Kalish, H., \& Bjorkavag, M. (2010). Energy Budget in a Dispersive Model for Undular Bores. Proceedings of the Estonian Academy of Sciences, 59, No. 2, 172 - 181. http://dx.doi.org/10.3176/proc.2010.2.17

Kang, H., Zhang, H., \& Qu, X. (2009). Numerical Study of Effect of Wave Around Single Break-Water with the Swan Model. Journal of Hydrodynamics, Ser. B. 21 (1), 136 - 141.

Khurmi, R. S. (2004). A Textbook of Hydraulics. (1 $1^{\text {th }}$ ed.). New Delhi: Schand and Company Ltd.

Pelinovsky, E. N., \& Rodin, A. A. (2011). Nonlinear Deformation of a Large-Amplitude Wave on Shallow Water. Doklady Physics, 56 (5), 305 - 308. http://dx.doi.org/10.1134/S1028335811050119 
Whitham, G. B. (1974). Linear and Nonlinear Waves. New York: John Wiley Interscience Publishers Inc.

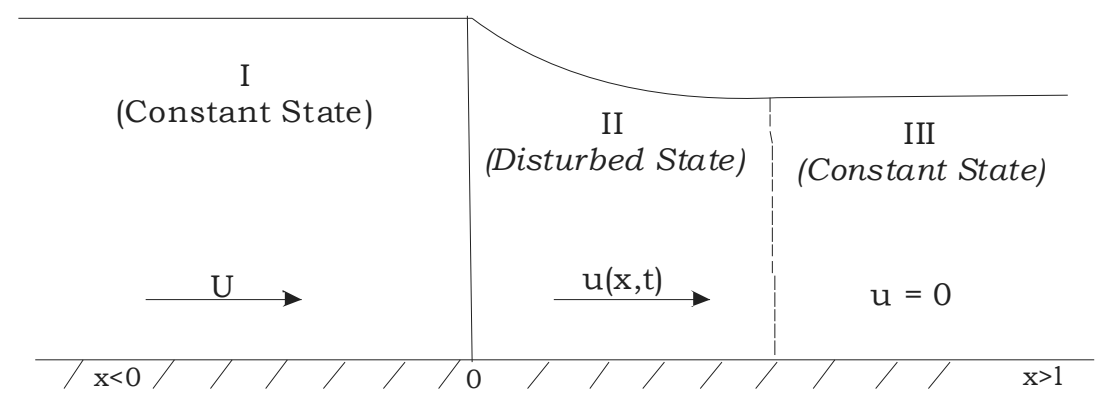

Figure 1. Flow in an open channel divided into three regions I, II, III

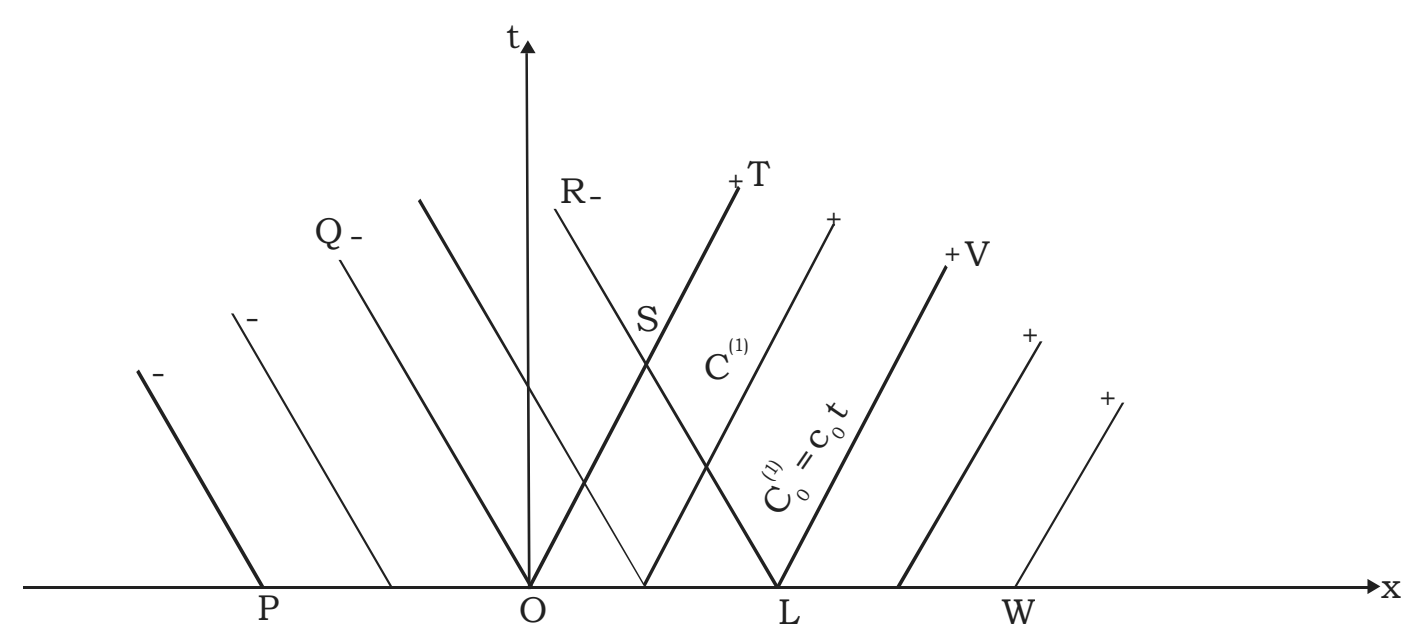

Figure 2. Definition sketch for positive $\mathrm{C}^{(1)}$ and negative $\mathrm{C}^{(2)}$ characteristics

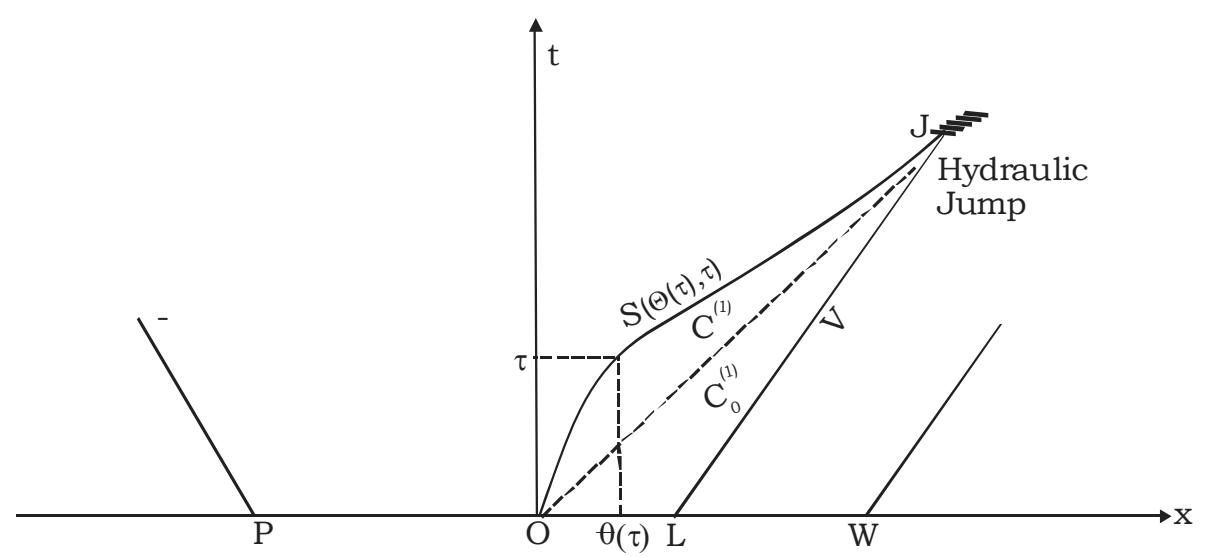

Figure 3. Formation of a hydraulic jump due to the intersecting characteristics ST and LV 